\title{
The Effect of Random Voids in the Modified Gurson Model
}

\author{
HUIYANG FEI, ${ }^{1}$ KYLE YAZZIE ${ }^{2}$ NIKHILESH CHAWLA,,${ }^{1,2,3}$ \\ and HANQING JIANG ${ }^{1,4}$
}

\begin{abstract}
1.-Mechanical and Aerospace Engineering, School for Engineering of Matter, Transport and Energy, Fulton Schools of Engineering, Arizona State University, Tempe, AZ 85287-8706, USA. 2.-Materials Science and Engineering, School for Engineering of Matter, Transport and Energy, Arizona State University, Tempe, AZ 85287-8706, USA. 3.—e-mail: nikhilesh.chawla@asu.edu. 4.-e-mail:hanqing.jiang@asu.edu
\end{abstract}

\begin{abstract}
The porous plasticity model (usually referred to as the Gurson-TvergaardNeedleman model or modified Gurson model) has been widely used in the study of microvoid-induced ductile fracture. In this paper, we studied the effects of random voids on the porous plasticity model. Finite-element simulations were conducted to study a copper/tin/copper joint bar under uniaxial tension using the commercial finite-element package ABAQUS. A randomly distributed initial void volume fraction with different types of distribution was introduced, and the effects of this randomness on the crack path and macroscopic stress-strain behavior were studied. It was found that consideration of the random voids is able to capture more detailed and localized deformation features, such as different crack paths and different ultimate tensile strengths, and meanwhile does not change the macroscopic stress-strain behavior. It seems that the random voids are able to qualitatively explain the scattered observations in experiments while keeping the macroscopic measurements consistent.
\end{abstract}

Key words: Gurson model, porous plasticity, random voids, finite-element analysis

\section{INTRODUCTION}

Nucleation, growth, and coalescence of microvoids in metallic materials are important mechanisms in ductile failure. ${ }^{1,2}$ In $\mathrm{Pb}$-free solder alloys, particularly Sn-rich solders with $\mathrm{Ag}$ and $\mathrm{Cu}$, second-phase particles of $\mathrm{Ag}_{3} \mathrm{Sn}$ and $\mathrm{Cu}_{6} \mathrm{Sn}_{5}$ are present. These particles, which can be as large as tens of micrometers or as small as submicrometer in size, have been shown to nucleate voids. ${ }^{3}$ Many investigators have conducted fractographic analyses that show a predominant distribution of voids, with the secondphase particles at the base of these voids. ${ }^{4}$ In other cases, voids can be nucleated close to $\mathrm{Cu}_{6} \mathrm{Sn}_{5}$ intermetallic compound (IMC) formed during reflow of Sn-rich solder and $\mathrm{Cu}$.

Therefore, theoretical descriptions and predictive numerical simulations of ductile failure due to

(Received March 26, 2011; accepted November 7, 2011; published online December 1, 2011) incubation and coalescence of microvoids are of great interest. In the $1960 \mathrm{~s}$, Rice and Tracey ${ }^{5}$ investigated ductile enlargement of a single void in an infinite matrix, and proposed that the void growth rate increases due to the hydrostatic stress $\sigma_{k k}$. In the $1970 \mathrm{~s}$, Gurson ${ }^{2}$ developed a porous plasticity model based on a unit cell with a single void in a finite matrix. Gurson ${ }^{2}$ established a yield criterion for porous ductile metals that depends on not only the von Mises effective stress $\sigma_{\mathrm{e}}$ (as in conventional plasticity) but also the hydrostatic stress $\sigma_{k k}$ and void volume fraction $f$. Later, Tvergaard and Needleman studied ductile crack growth based on the Gurson model, and further developed and modified this model to account for plastic work hardening and damage evolution. ${ }^{6-11}$ This porous plasticity model is referred to as the GursonTvergaard-Needleman (GTN) model or modified Gurson model, being widely used to model microvoid-induced ductile fracture. The GTN model has been included in the commercial finite-element 
package ABAQUS as one of the plastic models to study the evolution of damage in metallic materials containing microvoids and to analyze ductile fracture. Recently, Wen et al. ${ }^{12,13}$ extended the GTN model to account for the effect of void size and observed that the yield surfaces for micrometer- and submicrometer-sized voids are significantly larger than given by the Gurson model.

Experimentally, the size and location of nucleated microvoids are randomly distributed; for example, Fig. 1 shows a single $\mathrm{Cu}-\mathrm{Sn}-\mathrm{Cu}$ joint tested in tension. The joint was made of $99.99 \%$ pure tin (Sn) solder and oxygen-free high-conductivity (OFHC) copper bars. The thickness of the pure Sn joint was $0.5 \mathrm{~mm}$, and the diameter of the cylindrical specimen was $6.35 \mathrm{~mm}$. Figure 1a is an optical micrograph of the side view of the joint before loading. Figure $1 \mathrm{~b}$ shows scanning electron microscopy (SEM) images of the fracture surfaces of the two specimens. The fracture surfaces on the left and right are opposing matching fracture surfaces. Clearly, the voids are distributed randomly. It is, thus, necessary to be able to simulate the behavior of $\mathrm{Pb}$-free solder alloys with random distribution of nucleating voids.

In this work, we studied the effect of a random distribution of voids using the modified Gurson model in the finite-element package ABAQUS. Random sets of initial void volume fractions $f$ with different random distribution, such as rectangular or Gaussian distributions, were assigned to elements. The effects of mean void volume fraction, $f$, its standard deviation, and the type of distribution were studied. It was found that capturing the randomness of the voids in the model gives more detailed and local information of void nucleation and may explain some of the scatter in ultimate strength and crack path observed experimentally.

\section{THE GTN MODEL}

The GTN model assumes a ductile metal matrix material either in fully dense form or with a small initial distribution of voids. The void volume fraction $f$ is defined as the volume of voids divided by the total volume of the porous material; $f=0$ describes

(a)

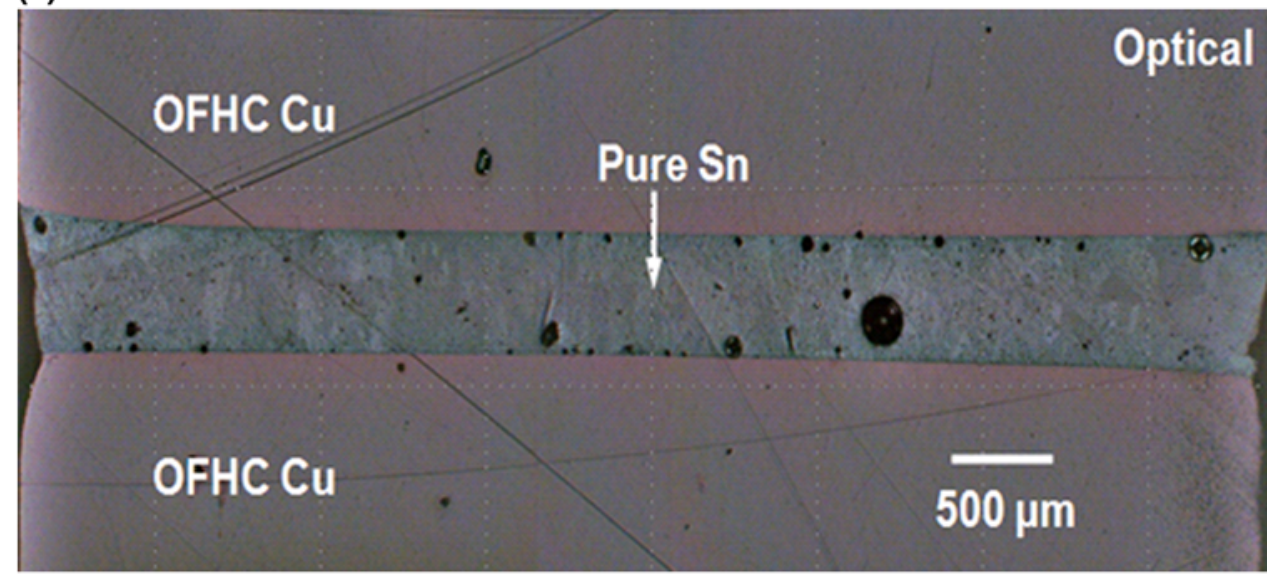

(b)

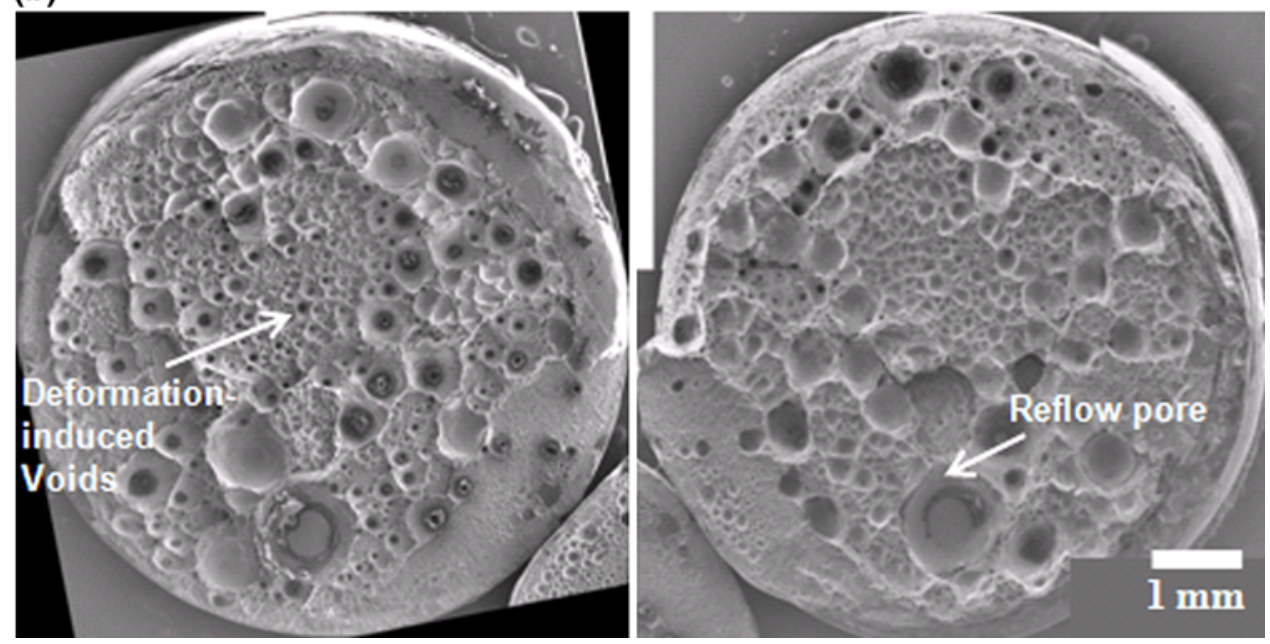

Fig. 1. (a) Optical image of the side view of a copper/tin/copper joint. (b) Scanning electron microscopy image of the crack surface of a copper/ tin/copper joint. 
a fully dense material, whereas $f=1$ represents a fully voided material. The GTN model proposes a yield surface given by ${ }^{2,6}$

$$
\phi=\left(\frac{\sigma_{\mathrm{e}}}{\sigma_{\mathrm{y}}}\right)^{2}+2 q_{1} f \cosh \left(-\frac{3 q_{2}}{2 \sigma_{\mathrm{y}}} \sigma_{\mathrm{kk}}\right)-\left(1+q_{3} f^{2}\right)=0,
$$

where $\sigma_{y}$ is the yield stress of the matrix material; the three parameters $\left(q_{1}, q_{2}\right.$, and $\left.q_{3}\right)$ were introduced by Tvergaard ${ }^{6}$ to make the original Gurson model agree with numerical studies. Tvergaard ${ }^{6}$ suggested that $q_{1}$ is dependent on the strain-hardening behavior of the metal; for example, $q_{1}=1.25$ for $n=20$ and $q_{1}=1.8$ for $n=5$, where $n$ is the strain-hardening exponent. It was also suggested that $q_{2}=1.0$ and $q_{3}=q_{1}^{2}$. Setting the void volume fraction $f=0$, the GTN collapses to the classical $J_{2}$ theory, which uses the von Mises yield criterion. Figure 2 shows representative yield surfaces expressed as the relationship between normalized von Mises effective stress and hydrostatic stress for $q_{1}=1.8, q_{2}=1.0$, and $q_{3}=3.24$. One can clearly see that the yield condition depends on both the von Mises effective stress and the hydrostatic stress, and with increasing void volume fraction, $f$, the material tends to yield at smaller von Mises stress and hydrostatic stress.

The evolution of the void volume fraction growth rate, $\dot{f}$, has two contributions, namely the nucleation rate and the growth rate,

$$
\dot{f}=\dot{f}_{\text {nucl }}+\dot{f}_{\text {gr }},
$$

where $\dot{f}_{\text {nucl }}$ changes due to nucleation of new voids and $\dot{f}_{\mathrm{gr}}$ changes due to growth of existing voids. Chu and Needleman ${ }^{8}$ assumed that the nucleation of new voids is plastic strain controlled and given by

$$
\dot{f}_{\text {nucl }}=A \dot{\bar{\varepsilon}}_{\mathrm{m}}^{\mathrm{pl}}
$$

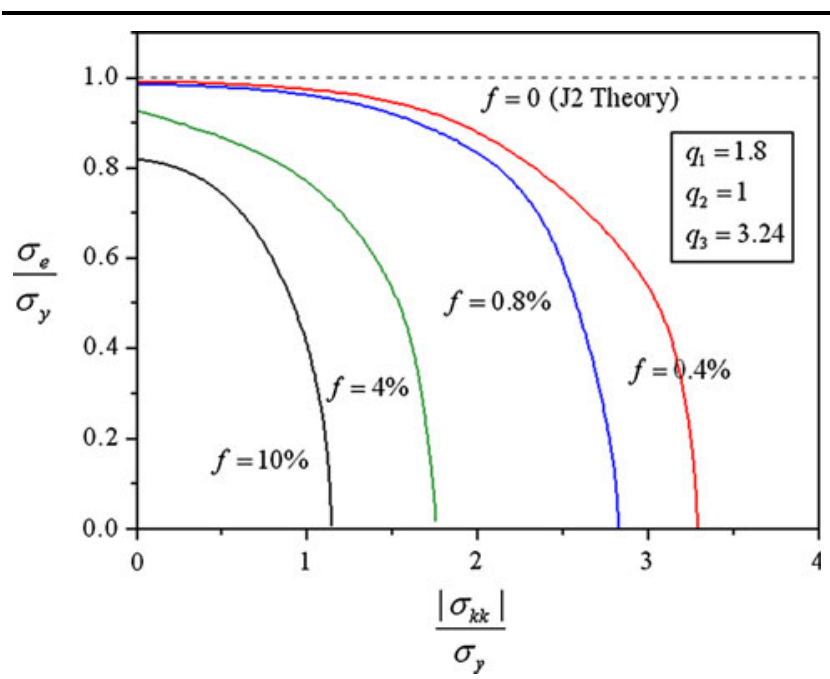

Fig. 2. Schematic of the yield surface of the modified Gurson model. where

$$
A=\frac{f_{\mathrm{N}}}{s_{\mathrm{N}} \sqrt{2 \pi}} \exp \left[-\frac{1}{2}\left(\frac{\bar{\epsilon}_{\mathrm{m}}^{\mathrm{pl}}-\varepsilon_{\mathrm{N}}}{s_{\mathrm{N}}}\right)\right]
$$

is a normal distribution and $\dot{\bar{\epsilon}}_{\mathrm{m}}^{\mathrm{p}}$ is the rate of equivalent plastic strain $\bar{\varepsilon}_{\mathrm{m}}^{\mathrm{pl}}$ of the material. Needleman and Rice ${ }^{14}$ suggested that void nucleation may take place over a narrow range of nucleation strain, characterized by mean $\varepsilon_{\mathrm{N}}$ and standard deviation $s_{\mathrm{N}}$. Usually, one can take $\varepsilon_{\mathrm{N}}$ as a strain value before the ultimate tensile strength (UTS) in the stress-strain curve. $f_{\mathrm{N}}$ is the upper limit of the total void volume fraction that can be nucleated, which is consistent with the volume fraction of second-phase particles of the metal. The void growth rate is given by

$$
\dot{f}_{\mathrm{gr}}=(1-f) \dot{\varepsilon}_{\mathrm{kk}}^{\mathrm{pl}}
$$

where $\dot{\varepsilon}_{\mathrm{kk}}^{\mathrm{pl}}$ is the rate of volumetric plastic strain of the material. Equation (4) was derived from the plastic incompressibility of the matrix material (not the material containing incompressible matrix and compressible voids).

\section{FINITE-ELEMENT ANALYSIS}

Compared with classical metal plasticity, the GTN model introduces a new state variable, the void volume fraction, $f$, into the constitutive relations. Therefore, the randomness of the voids can be implemented via random assignment of the initial void volume fraction to each element. The randomly assigned initial void volume fraction obeys a certain type of random distribution, such as rectangular or Gaussian. To handle a large number of elements, a $\mathrm{C}++$ code was developed to generate and assign random initial void volume fractions to each element, based on the prescribed distribution of initial voids.

Figure $3 \mathrm{a}$ illustrates the model problem and the boundary conditions. The model problem consists of a copper/tin/copper joint which reproduces the joint experiment shown in Fig. 1 . The $\mathrm{Sn}$ is $0.5 \mathrm{~mm}$ thick and $6.35 \mathrm{~mm}$ in diameter. Axisymmetric elements are used, namely CAX4R (four-node axisymmetric element with reduced integration) for copper and CAX3 (three-node axisymmetric element) for Sn. The symmetric boundary conditions were applied in the symmetry axes, and the bottom of the copper had fixed displacement in the vertical direction. The prescribed displacement boundary condition was applied at the top of the copper in the vertical direction. Mesh convergence was examined by varying the element numbers of pure $\mathrm{Sn}$, and finally the simulations used 8000 CAX3 elements in pure $\mathrm{Sn}$. All simulations were quasistatic with applied displacement.

Copper was modeled as an elastic material with Young's modulus of $E=116.5 \mathrm{GPa}$ and Poisson's ratio of $v=0.34$. Sn was modeled as a GTN plastic 
(a)
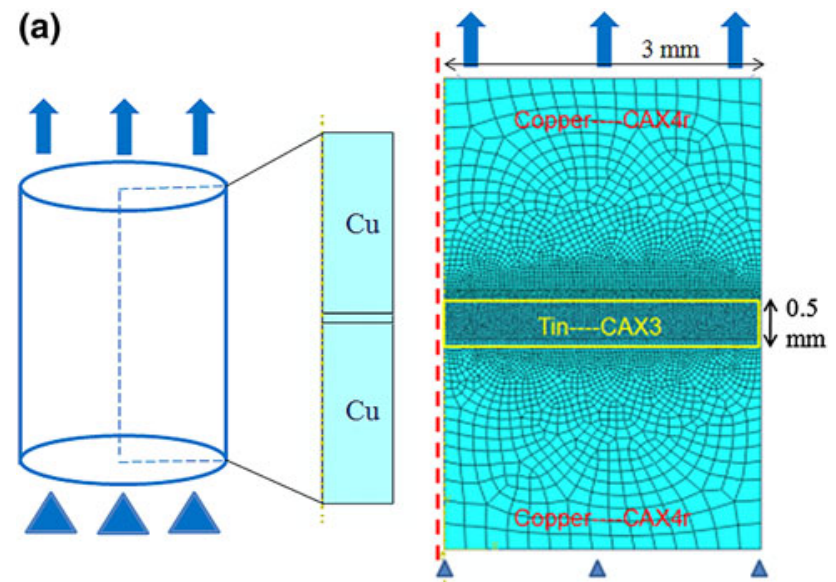

(b)

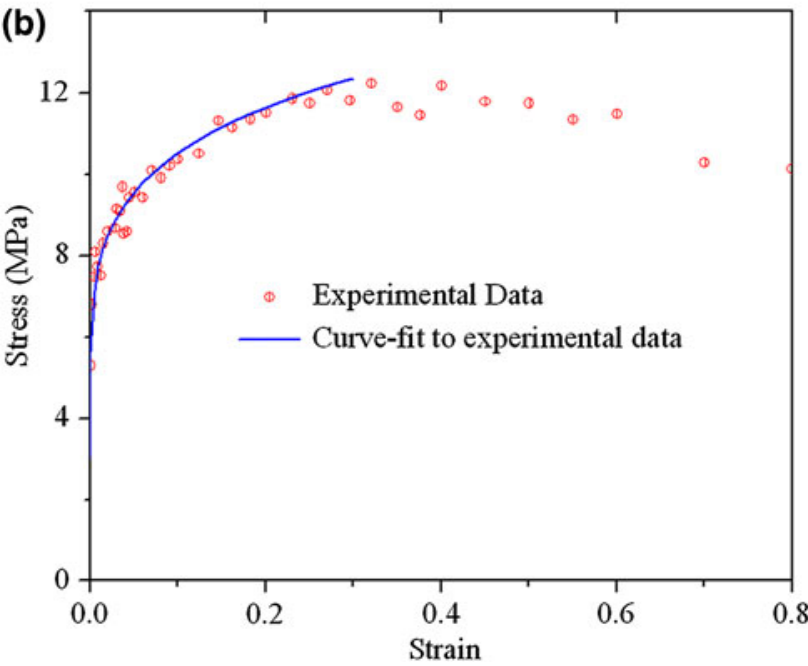

Fig. 3. (a) Geometry and boundary conditions of the axisymmetric model for the simulation. (b) Stress-strain curve measured for pure $\mathrm{Sn}$ at strain rate of $0.001 / \mathrm{s}$.

material with random initial void volume fraction. The elastic modulus of $\mathrm{Sn}^{15,16}$ is $E=46.9 \mathrm{GPa}$ and its Poisson's ratio is $v=0.36$. The experimentally measured stress-strain curve of pure tin was used for the plastic portion of the curve, as given in Fig. 3b. To best fit the stress-strain curve, the strain-hardening index was obtained as $n=5$. In the simulation, we only used the hardening part up to the ultimate tensile stress (UTS). The three parameters in the yield surface (Eq. (1)) were taken as $q_{1}=1.8, q_{2}=1.0$, and $q_{3}=3.24$ for $n=5$ based on Tvergaard. ${ }^{6}$ The mean value of the nucleation strain was taken as $\varepsilon_{N}=0.1$ (less than the strain at the UTS of 0.3), and the standard deviation was chosen as $s_{\mathrm{N}}=0.02$; the upper limit of the total void volume was taken as $f_{\mathrm{N}}=0.01$. The initial void volume fraction is on the order of that observed experimentally. It must be noted that, although the aforementioned parameters are empirical, the simulation results based on these empirical values qualitatively captured the failure process of a pure Sn joint.

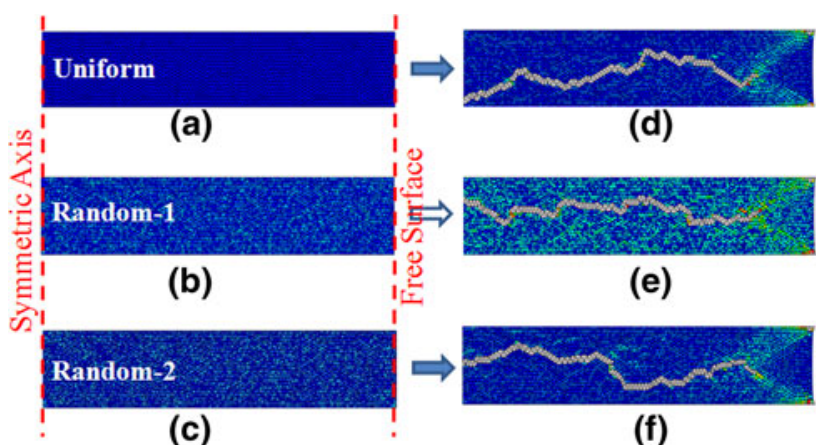

Fig. 4. Dependence of crack path on the randomness of the initial void volume fraction. Contours are for void volume fraction.

Here our main interest is to characterize the stress-strain response of the Sn segment. The following methods were used to calculate the stress and strain in Sn: Before necking, the stress state is uniaxial, so that the nominal normal stress $\sigma$ was calculated as the reaction force at the loading point (i.e., top of the copper) divided by the original crosssectional area. The strain in Sn was calculated as the height change of the Sn part divided by the original height of the Sn.

\section{RESULTS AND DISCUSSION}

First, we studied the effect of the initial void volume fraction on the fracture path in $\mathrm{Sn}$. We compared three situations as shown in Fig. 4, in which the contours indicate the void volume fraction. Figure 4a shows a case with a uniform initial void volume fraction throughout all elements in the Sn segment (Fig. 4a), and Fig. 4b, c shows two cases with randomly distributed initial void volume fractions in Sn. The initial void volume fraction was taken as $f=0.8 \%$, and the range of variation of the initial void volume fraction for the randomly distributed voids was from $0.3 \%$ to $1.3 \%$. A rectangular distribution was used to generate the randomly distributed initial voids for Fig. 4b, c. The randomness of the initial void volume fraction can be observed in Fig. 4b, c. After deformation, the three cases exhibited different fracture paths as shown in Fig. $4 d-f$. Because of the randomly distributed initial void volume fraction, the localizations of void nucleation and growth were also random, which led to different fracture patterns. Figure 5 compares the macroscopic stress-strain response of the Sn segment for the three cases in Fig. $4 \mathrm{a}-\mathrm{c}$. It was found that almost identical macroscopic stressstrain responses (both hardening and softening) were obtained for the three cases (Fig. $4 a-c$ ). This suggests that, by considering the randomly distributed voids in the GTN model, local microstructural effects that control the fracture path can be gleaned, while the macroscopic stress-strain behavior remains unchanged. It should be noticed that the softening segments of the output curves result from the increase of the void volume fraction, since the 


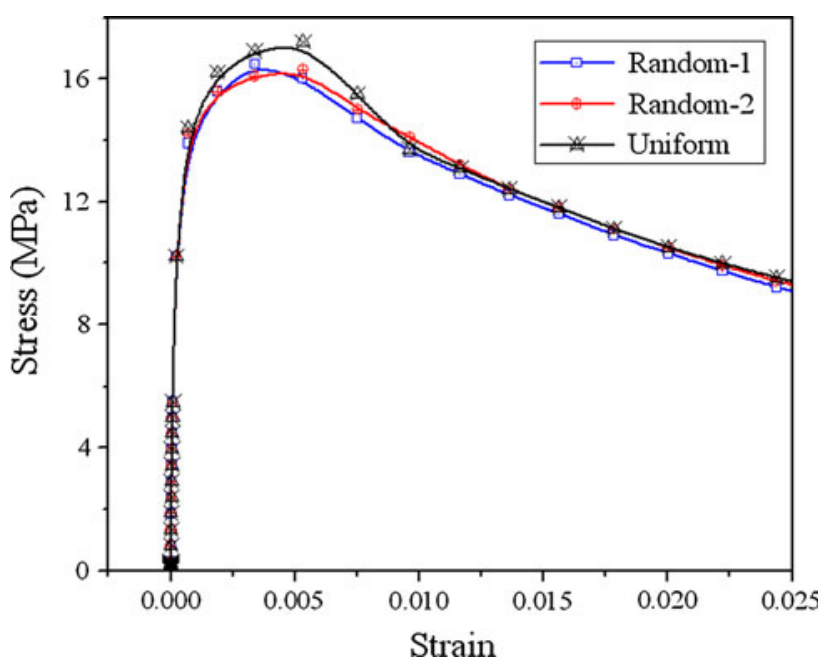

Fig. 5. Macroscopic stress-strain behavior of the tin segment with uniform and randomly distributed voids.

input has only the hardening segment (as shown in Fig. 3b). This observation agrees qualitatively with experiments in which different specimens prepared under identical conditions may have different cracking paths but exhibit very similar macroscopic stress-strain curves.

Secondly, we studied the effect of the range of the rectangular distribution of the initial void volume fraction on the macroscopic stress-strain behavior. Figure 6 shows three cases that have the same average initial void volume fraction of $0.8 \%$ but different ranges of rectangular distribution of $0 \%$ to $1.6 \%, 0.3 \%$ to $1.3 \%$, and $0.6 \%$ to $1.0 \%$, along with a uniformly distributed initial void volume fraction of $0.8 \%$. It was found that the different ranges did not affect the overall stress-strain curve but did affect the UTS. Specifically, the uniform case had the highest UTS while the case with the widest range (i.e., $0 \%$ to $1.6 \%$ ) showed the smallest UTS. This is because the overall stress-strain behavior is determined by the total void content in the material. Thus, all four cases, regardless of the distribution, exhibited similar behavior. It should be noted that a much larger range of voids may have elicited different results, i.e., have had a more profound effect on the deformation behavior. However, the UTS of the material is determined by the localization of the voids: Larger voids give smaller UTS, which explains why the case with the widest range of initial void volume fraction had the smallest UTS.

Thirdly, we studied the effect of the different types of random distribution on the macroscopic stress-strain behavior. Figure 7 shows the stressstrain curve for two types of distributions, namely rectangular and Gaussian. The average initial void volume distribution was taken as $0.8 \%$, the range for the rectangular distribution is $0.3 \%$ to $1.3 \%$, and the standard deviation of the Gaussian distribution is 0.02 . The results suggest that the macroscopic stress-strain behavior is not sensitive to the distri-

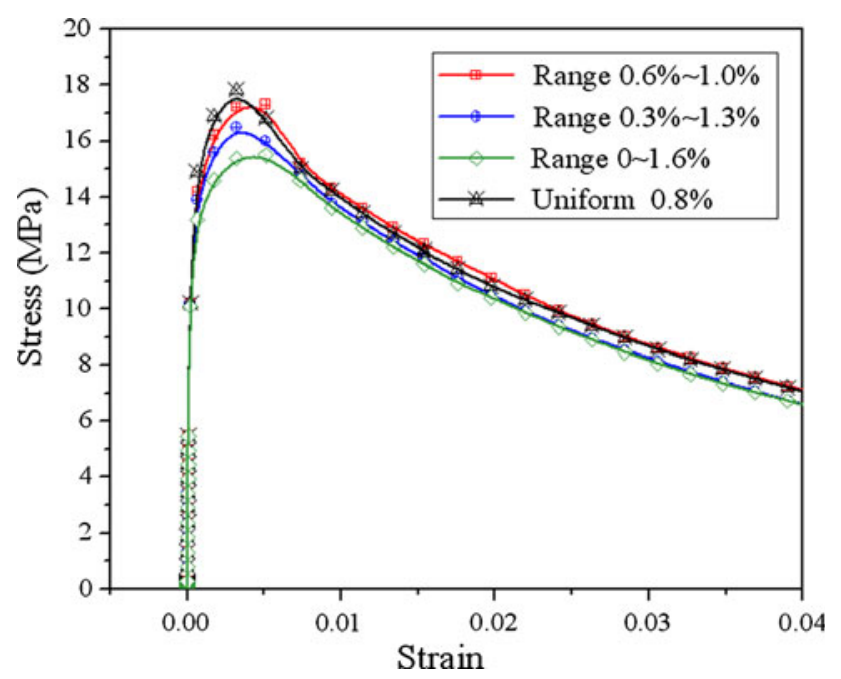

Fig. 6. Effect of the range of random distribution on the macroscopic stress-strain behavior.

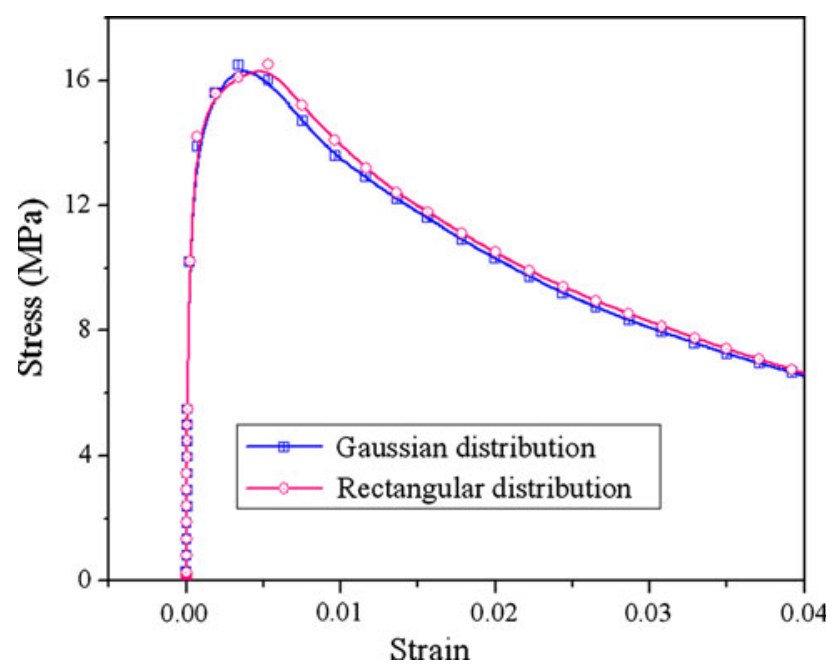

Fig. 7. Effect of the type of random distribution on the macroscopic stress-strain behavior.

bution types, which is similar to the observation of Fig. 6, i.e., that the macroscopic stress-strain behavior is mainly determined by the total voids of the materials. More explicitly, the macroscopic stress-strain behavior represents the overall loadbearing capability, which is related to the total fraction of voids. Since the two distributions have comparable average and maximum void volume fraction, the macroscopic stress-strain behaviors are similar.

Finally, we want to discuss a common phenomenon relating to the crack path that was found in all the aforementioned simulations. We found that all cracks initiated at the symmetry of axes and propagated towards the free surface of the cylindrical bar, even though the crack paths were different. As the tensile load increases, the stress and the plastic strain in the Sn segment increase, which in turn 


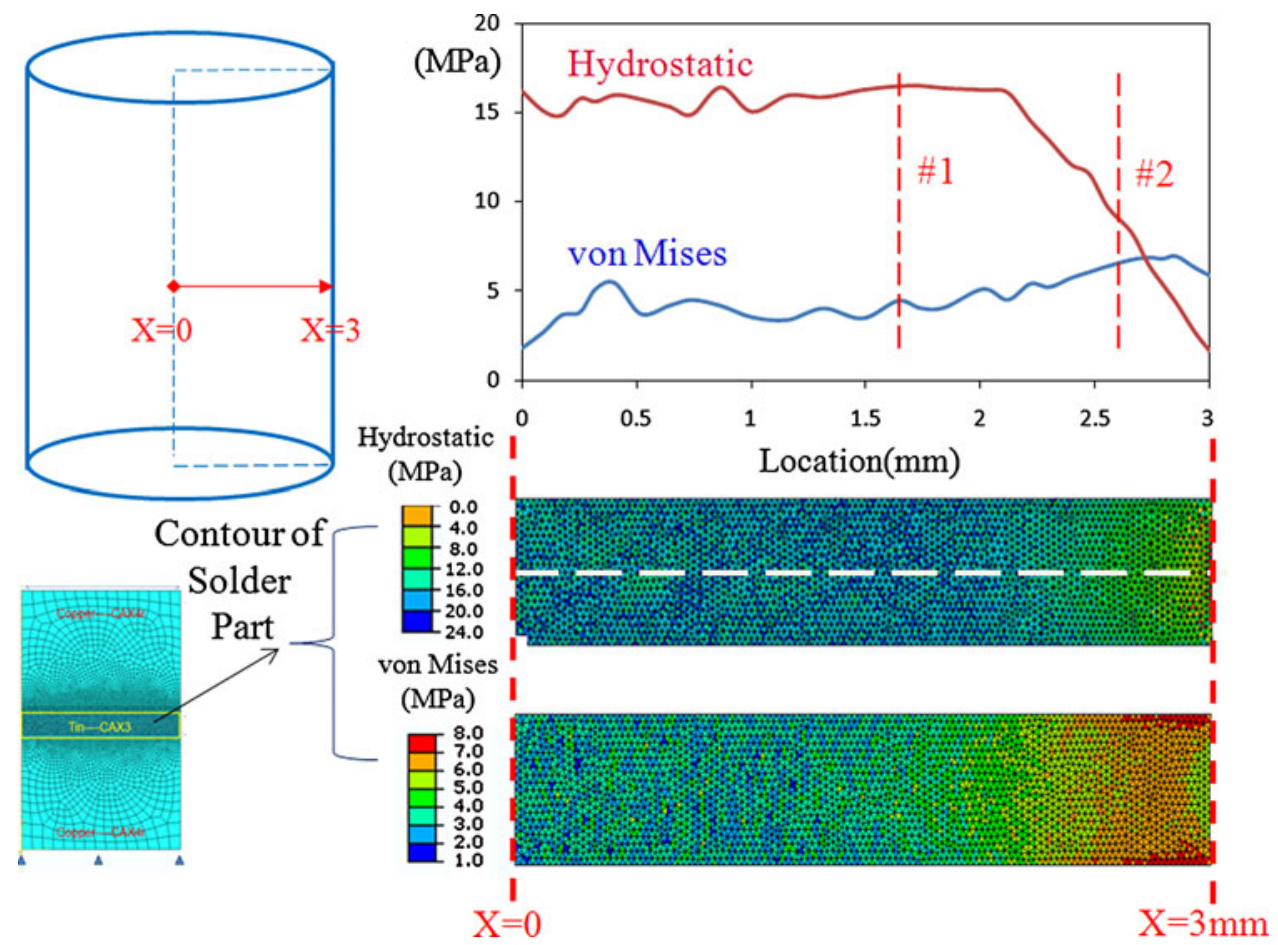

Fig. 8. Variation of hydrostatic stress and von Mises effective stress along the radial direction of the pure Sn segment.

leads to increase of the void volume fraction. In the yield surface given by Eq. (1), for given void volume fraction, the yield condition is determined by two quantities, namely the von Mises effective stress $\sigma_{\mathrm{e}}$ and the hydrostatic stress $\sigma_{k k}$. Here, compressive hydrostatic stress is considered negative and hydrostatic stress under tension is defined as positive. Figure 8 shows the von Mises effective stress $\sigma_{\mathrm{e}}$ and the hydrostatic stress $\sigma_{k k}$ in the Sn segment along the radial direction. The von Mises stress along the radial direction does not change significantly. On the contrary, the hydrostatic stress drops significantly in the vicinity of the free surface. Based on the yield surface given by Fig. 2, the elements close to the symmetry axis (with larger hydrostatic stress) will yield first, followed by the elements close to the free surface (with smaller hydrostatic stress). Once the element yields, the void volume fraction starts to increase, as characterized by Eqs. (2)-(4). Fracture occurs once the void volume fraction reaches a critical value.

\section{CONCLUSIONS}

In this work, we studied the effects of random voids on the porous plasticity model, i.e., the modified GTN model. Finite-element simulations were conducted using the commercial finite-element package ABAQUS. We introduced randomly distributed initial void volume fraction with different types of distribution and then studied the effects of this randomness on the crack path and macroscopic stress-strain behavior. We found that this consid- eration of the random voids is able to capture more detailed and localized deformation features, such as different crack paths and different UTS values, and meanwhile does not change the macroscopic stressstrain behavior. It seems that the random voids are able to qualitatively explain the scattered observations in experiments while keeping the macroscopic measurement consistent.

\section{ACKNOWLEDGEMENTS}

The authors are grateful for financial support for this work from the National Science Foundation, Division of Materials Research-Metals Division, DMR-0805144 (Drs. Allan Ardell, Bruce Macdonald, and Harsh Chopra, Program Managers). We also appreciate the Fulton High Performance Computing at Arizona State University for enabling us to conduct our simulations.

\section{REFERENCES}

1. J. Gurland and J. Plateau, Trans. Am. Soc. Met. 56, 443 (1963).

2. A.L. Gurson, J. Eng. Mater. Technol. Trans. ASME 99, 2 (1977).

3. X. Deng, R.S. Sidhu, P. Johnson, and N. Chawla, Metall. Mater. Trans. A 36A, 55 (2005).

4. F. Ochoa, J.J. Williams, and N. Chawla, JOM 55, 56 (2003).

5. J.R. Rice and D.M. Tracey, J. Mech. Phys. Solids 17, 201 (1969).

6. V. Tvergaard, Int. J. Fract. 17, 389 (1981).

7. V. Tvergaard, J. Mech. Phys. Solids 30, 399 (1982).

8. C.C. Chu and A. Needleman, J. Eng. Mater. Technol. Trans. ASME 102, 249 (1980).

9. V. Tvergaard, Int. J. Solids Struct. 18, 659 (1982).

10. V. Tvergaard, Int. J. Fract. 18, 237 (1982). 
11. A. Needleman and V. Tvergaard, Eng. Fract. Mech. 38, 157 (1991).

12. J. Wen, Y.G. Huang, and K.C. Hwang, Acta. Mech. Sinica 20, 393 (2004).

13. J. Wen, Y. Huang, K.C. Hwang, C. Liu, and M. Li, Int. J. Plast. 21, 381 (2005).
14. A. Needleman and J.R. Rice, Mechanics of Sheet Metal Forming (New York: Plenum, 1978).

15. X. Deng, N. Chawla, K.K. Chawla, and M. Koopman, Acta Mater. 52, 4291 (2004).

16. X. Deng, M. Koopman, N. Chawla, and K.K. Chawla, Mater. Sci. Eng. 364, 240 (2004). 